\title{
L'intentionnalité des émotions : du corps aux valeurs
}

Julien A. Deonna et Fabrice Teroni

\section{OpenEdition}

1 Journals

Édition électronique

URL : http://journals.openedition.org/ress/61

DOI : $10.4000 /$ ress.61

ISSN : 1663-4446

Éditeur

Librairie Droz

Édition imprimée

Date de publication : 1 mai 2009

Pagination : 25-41

ISBN : 978-2-600-01303-1

ISSN : 0048-8046

Référence électronique

Julien A. Deonna et Fabrice Teroni, «L'intentionnalité des émotions : du corps aux valeurs », Revue européenne des sciences sociales [En ligne], XLVII-144 | 2009, mis en ligne le 01 mai 2012, consulté le 30 avril 2019. URL : http://journals.openedition.org/ress/61 ; DOI : 10.4000/ress.61 


\section{L'INTENTIONNALITÉ DES ÉMOTIONS : DU CORPS AUX VALEURS*}

Lorsque l'on considère les émotions indépendamment de toute entreprise théorique, deux constats s'imposent. D'un côté, les émotions possèdent une dimension phénoménale ou expérientielle: il y a un «effet que cela fait» que de ressentir une émotion, et cet effet se manifeste de manière particulièrement saillante dans la conscience des perturbations physiologiques propre aux expériences émotionnelles fortes. De l'autre, les émotions nous mettent en lien avec notre environnement et plus spécifiquement avec certains de ses aspects: ressentir une émotion, n'est-ce pas réagir à certains traits saillants des situations auxquelles nous faisons face? Nous avons peur de tel homme peu avenant, nous sommes indignés par telle remarque ou soulagés de retrouver notre portefeuille. C'est là la dimension intentionnelle des émotions. Il n'est pas étonnant, dès lors, que les théories des émotions se soient toujours distinguées précisément par l'importance respective qu'elles accordent à ces deux dimensions. En effet, il est notoirement difficile de les articuler de manière satisfaisante au sein d'une théorie des émotions. Pourquoi? Les perturbations physiologiques liées aux émotions ont été bien souvent citées pour affirmer qu'elles se situent au mieux en dehors du champ de la rationalité, au pire dans une relation conflictuelle avec celui-ci. Il est cependant aujourd'hui courant de souligner le rôle important des émotions dans l'exercice de nos facultés rationnelles ${ }^{1}$. Est-ce à dire que la dimension intentionnelle des émotions qui, nous allons le voir, permet de parler d'une rationalité au moins minimale dans le domaine des émotions, doit être conçue comme indépendante de leur dimension corporelle? La réponse à cette question est délicate, car si de nombreuses théories contemporaines insistent sur la dimension intentionnelle des émotions sans nier leur dimension phénoménologique, elles sont rarement explicites quant aux liens entre intentionnalité et phénoménologie d'une part, entre phénoménologie et corps ressenti de l'autre ${ }^{2}$. Notre objectif dans cet essai consiste à montrer qu'il est possible de comprendre l'intentionnalité des émotions, et donc ce qui permet de parler d'une rationalité affective, en terme de phénoménologie corporelle.

* Nous tenons à remercier pour leur soutien les membres du groupe de recherche Thumos et le Pôle National de Recherche $(\mathrm{CH})$ en Sciences Affectives.

1 Ces rôles dans la rationalité ont naturellement été conçus de manières très différentes. Pour apprécier ces différentes approches, voir notamment Damasio (1994), De Sousa (1987), Jones (2006) et, en particulier, Livet (2002).

2 En particulier, s'il est aujourd'hui courant de souligner la nécessité d'articuler de manière satisfaisante ces deux dimensions (e.g. Deonna (2006), Döring (2007), Goldie (2000)), ceci est rarement suivi d'analyses détaillées de ce problème. 


\section{LES ÉMOTIONS COMME ACCÈS AUX VALEURS}

Commençons par considérer la dimension intentionnelle des émotions. Nous sommes rapidement amenés à dresser le constat suivant. Si l'intuition que nous soulignions plus haut consiste à affirmer que les émotions réagissent à certains traits saillants et semblent porter intentionnellement vers ceux-ci, les traits pertinents se laissent aisément comprendre en termes évaluatifs. Dans le cas de la honte, par exemple, celles-ci sont à élucider en terme de dégradation de soi, dans la tristesse en terme de perte, dans l'admiration en terme de valeurs morales ou esthétiques, et dans la peur en terme de danger. Ce lien intime entre émotions et propriétés évaluatives est d'autant plus frappant lorsque l'on constate que nombre de termes évaluatifs courants sont directement dérivés du langage des émotions, comme le dégoûtant, l'amusant, le regrettable, etc. ${ }^{3}$

A partir de ce constat, nous sommes naturellement conduits à penser que les émotions nous mettent en lien avec des valeurs. Qu'est-ce que cela signifie? Il nous faut d'abord spécifier la notion pertinente de valeur et ensuite élucider quelque peu l'idée selon laquelle les émotions nous mettent en relation avec les valeurs. En ce qui concerne la première question, les philosophes qui ont développé cette idée parlent de valeurs dans un sens quelque peu différent de celui que le terme revêt dans le langage ordinaire. Les valeurs ne sont en effet pas considérées comme des objets abstraits ou idéaux vers lesquels nous tendons, il s'agit plutôt de propriétés d'un type particulier que des situations, des objets ou des comportements exemplifient: les émotions nous mettraient en lien avec des exemplifications de ces propriétés évaluatives. Par ailleurs, comme les exemples l'indiquent déjà clairement, une valeur peut être soit positive (le beau, le courageux, le juste), soit négative (le danger, la vulgarité, la perte). En ce qui concerne la seconde question, l'idée fondamentale est la suivante. $\mathrm{Si}$, pour prendre un exemple très simple, nos sens nous permettent d'appréhender par la vue la forme et les couleurs de ce chien, ainsi que par l'ouïe les sons qu'il émet, les émotions nous permettent quant à elles d'appréhender les propriétés évaluatives qu'il exemplifie. Affirmer qu'il existe une relation intentionnelle entre émotions et valeurs revient à dire, donc, que les différents types d'émotions constituent autant de modes d'appréhension de différents types de valeurs. Ainsi, la peur serait un mode d'appréhension du danger, la colère un mode d'appréhension de l'offense, etc.

Une telle approche possède sans aucun doute un caractère intuitif, mais il convient maintenant de s'interroger sur ses avantages théoriques. C'est qu'elle permet d'envisager des réponses séduisantes à certaines questions fondamentales que toute théorie des émotions se doit de considérer. Faire appel aux valeurs, selon les défenseurs de cette approche, fournit en effet le moyen d'individuer, de rendre intelligibles et d'évaluer les émotions et, par ce biais, d'ouvrir la porte à une forme de rationalité affective. Cela permet de les individuer dans la mesure où l'appel à une valeur spécifie ce qui unifie les différentes occurrences d'une émotion d'un même type. Si des occurrences d'un même type d'émotion (e. g. la tristesse) peuvent porter sur différents objets particuliers (e. g. la mort de mon chat, mon

3 Voir en particulier Mulligan (1998) et Roberts (2003), qui propose une typologie des émotions fondée sur leurs liens aux valeurs. 
échec à l'examen, la faillite de ma banque), cette approche suggère qu'elles n'en partagent pas moins le fait qu'elles portent toutes sur une même valeur (e. g. le sujet appréhende ces différents objets comme des pertes). Les types d'émotions sont ainsi unifiés au niveau de leur intentionnalité évaluative. Ce recours aux valeurs autorise ensuite à rendre intelligibles ou non les réponses affectives d'un sujet. Nous comprenons en effet pourquoi des situations radicalement différentes suscitent la même émotion lorsque nous saisissons le fait qu'elles exemplifient ou paraissent aux yeux du sujet exemplifier la même valeur. C'est en ce sens qu'une émotion est intelligible, ou au contraire inintelligible lorsqu'il nous est impossible de comprendre comment la situation peut être saisie par le sujet comme exemplifiant une valeur donnée. Le recours aux valeurs permet enfin d'évaluer les émotions. Nous considérons couramment que certaines occurrences d'émotions sont appropriées ou inappropriées. Au sein de l'approche en question, ce contraste doit être compris en terme de l'exemplification ou non de la valeur par la situation vers laquelle l'émotion est dirigée. Si une remarque ne constitue en rien une offense, on considère alors la colère qu'elle suscite comme inappropriée. On parle souvent pour cette raison des conditions de correction évaluatives des émotions ${ }^{4}$. Ce sont ces rôles fondamentaux joués par les valeurs que les philosophes ont à l'esprit lorsqu'ils y font référence en tant qu'objets formels des émotions ${ }^{5}$. Et, dans la mesure où l'on distingue ainsi les émotions intelligibles et appropriées de celles qui ne le sont pas, on commence à envisager le sens dans lequel les émotions sont sujettes à des contraintes normatives et rationnelles. Mais, si rendre compte de la rationalité des émotions ainsi comprise présente de nombreux avantages, que peut bien signifier, au niveau psychologique, l'idée selon laquelle les émotions sont des modes d'appréhension de valeurs?

Une manière traditionnelle de concevoir ces modes d'appréhension, familière depuis l'antiquité, consiste à définir les émotions comme des jugements de valeur ${ }^{6}$. Etre en colère, c'est juger que l'on est offensé, se sentir coupable juger que l'on a commis une faute, avoir peur juger que l'on fait face à un danger. Souscrire à une telle définition permet en effet, et c'est là son avantage principal, de respecter les rôles joués par les valeurs dans l'économie affective. Cependant, si la référence au jugement est prise au sérieux, la prétention de l'analyse à parler des émotions est mise à mal pour deux raisons principales. Premièrement, nous avons de solides raisons de douter de la nécessité d'un jugement évaluatif pour l'occurrence d'une émotion. Si des créatures incapables de pensées évaluatives, à l'instar de certains animaux et des jeunes enfants, peuvent néanmoins ressentir des émotions -et cela ne fait aucun doute, alors les émotions ne sauraient être des

4 Tout comme la perception visuelle qui me présente une table brune à tel endroit possède des conditions de correction (la présence d'une table de telle couleur en telle position), une émotion de peur qui me présente ce chien comme dangereux possède des conditions de correction évaluatives (la présence d'un chien dangereux). Dans les deux cas, si le monde ne correspond pas à la manière dont il m'est présenté, on parle de représentation erronée.

5 Pour l'introduction de la notion d'objet formel en rapport avec les émotions, voir Kenny (1963). Pour les différents rôles joués par les objets formels, voir Teroni (2007).

6 Cette théorie défendue par les Stoïciens a été remise au goût du jour par Nussbaum (2001) et Solomon (1988). 
jugements ${ }^{7}$. Deuxièmement, l'analyse ne saurait en outre être suffisante, dans la mesure où il n'est pas rare d'émettre de tels jugements sans être sous l'emprise de l'émotion que l'on cherche à analyser. Il doit y avoir une différence entre juger qu'il y a du danger à vivre à Los Angeles et avoir peur d'y vivre, ce dont la théorie ne peut rendre compte.

C'est principalement pour ces raisons qu'il est aujourd'hui convenu d'admettre que, s'il y a appréhension de valeurs au sein des émotions, celle-ci ne saurait être comprise sur le modèle du jugement. Et ce que l'objection de la suffisance met bien en lumière, c'est naturellement le fait qu'une analyse satisfaisante des émotions doit certes rendre justice à leur dimension intentionnelle, mais ne pas négliger ce faisant leur aspect phénoménal; car c'est bien la présence d'une phénoménologie particulière des émotions qui rend impossible leur assimilation aux jugements ${ }^{8}$. De plus, l'analyse doit combiner ces deux aspects sans pour autant tomber dans le vice propre aux analyses des émotions en termes purement phénoménaux, qui pêchent en refusant d'admettre que c'est en vertu de leur intentionnalité, c'est-à-dire du fait qu'elles pointent au-delà d'elles-mêmes, que les émotions sont aptes à jouer un rôle dans la négociation par le sujet des opportunités et défis que son environnement lui présente. Respecter ces deux aspects est ce que nous promet le modèle perceptif des émotions vers lequel nous nous tournons maintenant.

\section{DU MODÈLE PERCEPTIF AU MODÈLE EXPÉRIENTIEL}

A la lumière du problème consistant à rendre compte des dimensions phénoménales et intentionnelles des émotions, le modèle qui a récemment trouvé faveur auprès de nombreux philosophes est celui de la perception ${ }^{9}$. L'idée revient simplement à concevoir les émotions non plus comme des jugements de valeur, mais comme des perceptions de celles-ci. Après tout, la perception sensorielle n'estelle pas précisément caractérisée par sa double nature, à la fois phénoménale et intentionnelle? De surcroît, il semble que, dans la perception, phénoménologie et intentionnalité ne soient pas indépendantes: à première vue au moins, nous sommes tentés de dire qu'il est difficile de séparer le fait que mon expérience a telles ou telles propriétés phénoménales (une expérience de rouge) du fait que cette expérience représente tel ou tel objet comme rouge. Si le défi consiste à combiner dans une même analyse les propriétés phénoménales et intentionnelles, et ainsi à combiner les avantages respectifs des théories judicatives et phénoménales des émotions sans en partager les défauts, on comprend dès lors l'attrait d'un modèle perceptif. Et ce dernier paraît séduisant à plus d'un titre. Au-delà des aspects communs entre perceptions et émotions déjà mentionnés, on doit également souligner les parallèles suivants.

7 Pour cette critique, voir notamment Deigh (1994) et Tappolet (2000).

8 On rencontre ici une critique similaire à celle fréquemment émise contre les théories qui cherchent à analyser la perception en terme de jugements, comme celle défendue par Armstrong (1968).

9 Ce modèle, anticipé par De Sousa (1987), est exploré en détail par Tappolet (2000) et défendu notamment par Prinz (2004), Deonna (2006) et Döring (2007). 
D'abord, perception et émotions sont considérées de manière plausible comme deux sources d'information sui generis à propos d'un domaine particulier de propriétés. Si nos sens constituent autant d'accès uniques ou privilégiés aux propriétés perceptives de notre environnement, les émotions sont dans le même rapport à ses propriétés évaluatives. Deuxièmement, un état perceptif, comme une émotion, est un état mental qu'il est possible d'instancier indépendamment de la maîtrise des concepts pertinents à l'articulation de son contenu. Un animal peut percevoir telle nuance de rouge, ou percevoir le danger auquel il fait face, sans pour autant maîtriser respectivement les concepts de rougeur et de danger nécessaires pour émettre les jugements pertinents ${ }^{10}$. Troisièmement et en lien direct avec cette dernière remarque, il existe un parallèle intéressant entre perception et jugement perceptif d'un côté, émotion et jugement évaluatif de l'autre. Tout comme il n'est pas rare que nos jugements perceptifs rejettent les verdicts de nos sens, il n'est pas rare non plus que nos jugements évaluatifs se désolidarisent de nos réponses affectives à notre environnement. Je juge que cet objet n'est pas bleu même s'il m'apparaît tel à la lumière de l'aube, je juge que cette couleuvre européenne ne présente pas le danger qui rendrait ma peur appropriée. L'avantage plus spécifique du modèle perceptif sur ce point consiste à admettre la présence de tels conflits sans pour autant attribuer d'irrationalité au sujet ${ }^{11}$. Enfin, l'existence du parallèle entre perception et jugement perceptif d'un côté, émotion et jugement évaluatif de l'autre contient la promesse d'une épistémologie séduisante dans le domaine des valeurs: s'il est plausible d'affirmer que nos états perceptifs causent et justifient nos jugements de perception, ne l'est-il pas tout autant de dire que nos jugements de valeur sont souvent causés et justifiés par nos émotions ${ }^{12}$ ? Si le recours au modèle perceptif était initialement motivé par l'espoir d'articuler phénoménologie et intentionnalité d'une manière éclairante, la constatation de l'existence de multiples et profonds parallèles pourrait laisser penser que nous nous trouvons face à plus qu'une simple analogie. Les émotions ne sont-elles pas simplement et littéralement des perceptions de valeurs?

Pour répondre à cette question, il nous faut à présent nous interroger sur la possibilité d'une application aussi littérale du modèle. Il existe en effet certaines disanalogies évidentes entre émotions et perceptions qui mettent en cause une assimilation aussi directe des premières aux secondes. Nous nous contenterons ici de souligner les principales. D'abord, si la perception dépend de l'activité d'organes sensoriels, il est loin d'être évident que parler d'un ou de plusieurs organes dans le cas des émotions fasse sens. Ensuite, les émotions possèdent une certaine polarité: elles sont soit positives soit négatives, ce qui ne fait à nouveau aucun sens dans le cas des perceptions ${ }^{13}$. En outre, si la perception semble fournir de

${ }^{10}$ Le débat entre conceptualistes et non conceptualistes a bien sûr divisé les philosophes de la perception. Pour une défense détaillée de la position non conceptualiste, voir en particulier Crane (1992).

${ }^{11}$ Pour un développement de ce point, voir Tappolet (2000) et Döring (2007).

${ }^{12}$ Ainsi, de même que, toutes choses étant égales, un état perceptif spécifique cause et justifie le jugement qu'un arbre se trouve devant moi, toutes choses étant égales une émotion de peur suscitée par ce chien cause et justifie le jugement que ce chien est dangereux.

${ }^{13}$ On parle souvent dans ce cadre de la valence des émotions. Sur ce concept, voir en particulier Colombetti (2005). 
l'information à propos de notre environnement de manière relativement indépendante de nos états motivationnels, on souligne souvent la haute dépendance de nos émotions par rapport à nos désirs, envies, humeurs et traits de caractère.

Enfin, deux disanalogies importantes dont nous aurons l'occasion de reparler en détail doivent également être soulignées. La première concerne ce que les philosophes ont aujourd'hui coutume d'appeler la transparence des états perceptifs ${ }^{14}$. Décrire nos expériences perceptives, par opposition à ce sur quoi elles portent, revient toujours en fait à décrire précisément ce sur quoi portent ces expériences. Décrire mon expérience lorsque je vois ce bol bleu et évasé ne peut que consister en une description des aspects bleuté et évasé que me présente le bol. La perception est transparente. Or, il est loin d'être clair que ce trait soit partagé par les expériences émotionnelles. En effet, décrire mon expérience affective lorsque je ressens de la peur à la vue de ce chien ne semble pas exclusivement consister en la description des aspects que me présente ce chien dangereux. La description de cette expérience fera bien plutôt allusion à ce que je ressens dans mon corps battements du cœur, sueurs froides, plutôt qu'aux diverses propriétés de l'objet sur lequel elle porte. En un mot, la phénoménologie du corps propre aux expériences affectives rend difficile l'application du principe de transparence dans le domaine des émotions.

La seconde disanalogie, la plus significative à nos yeux, provient du fait que si la perception constitue un accès indépendant à l'information à laquelle celle-ci nous rend sensible, cela n'est pas le cas des émotions. En effet, même si elles constituent un accès sui generis à des propriétés évaluatives de l'environnement, les émotions ne peuvent pas y accéder indépendamment d'autres bases cognitives (comme une croyance, un souvenir, ou même précisément une perception). Pour avoir peur de tel chien, par exemple, il faut le voir ou avoir une croyance à son propos. Cette disanalogie est cruciale dans la mesure où l'une des tâches centrales d'une théorie des émotions consiste précisément à articuler le type de relations existant entre les émotions et leurs bases cognitives. Il est par exemple fort plausible d'affirmer que certaines perceptions causent et justifient certaines émotions ${ }^{15}$, une thèse qu'il devient difficile de défendre si les émotions sont directement assimilées à des perceptions.

Le trajet effectué jusqu'ici doit nous mener à la conclusion suivante. L'analogie entre émotion et perception est fructueuse, mais elle ne doit pas être prise littéralement. Fondre les émotions dans le moule de la perception conduit à omettre des différences majeures entre ces deux types d'états mentaux. En revanche, l'analogie met le doigt sur des parallèles instructifs, et de ce fait révèle un certain nombre de contraintes qui pèsent sur toute théorie des émotions. Plus spécifiquement, une théorie qui cherche à combiner les dimensions intentionnelles et phéno(2008).

${ }^{14}$ Sur cette notion, voir par exemple Tye (2002) et, pour son application aux émotions, Tye

${ }^{15}$ Les liens de causalité et de justification dans le domaine des émotions sont donc complexes. Il ne faut en effet pas confondre les liens existant entre émotions et jugements évaluatifs (voir note 12) et ceux que nous mentionnons ici. Ces derniers concernent la relation entre base cognitive et émotion, par exemple entre la perception visuelle de ce chien tous crocs dehors et la peur qu'elle peut causer et justifier. Sur cette question, voir Goldie (2004) et Teroni (2007). 
ménales des émotions - ce que nous appellerons désormais une théorie expérientielle - doit satisfaire trois contraintes directement dévoilées par l'analogie entre émotions et états perceptifs. La première est la contrainte intentionnelle. Celle-ci stipule simplement que les émotions présentent au sujet qui en fait l'expérience certaines propriétés évaluatives de son environnement. La deuxième est la contrainte phénoménologique. Ici, l'idée est non seulement que la présentation des propriétés évaluatives que fournissent les émotions possède une dimension phénoménale, mais que ces propriétés phénoménales ont un rôle direct à jouer dans la représentation des propriétés évaluatives avec lesquelles ces émotions nous mettent en rapport. Il nous paraît en effet fort plausible - et cela consiste à prendre l'analogie avec la perception au sérieux - d'affirmer que les propriétés phénoménales des émotions jouent un rôle fondamental dans leur fonction représentationnelle. En effet, de même que ce n'est pas un hasard si le rouge nous est présenté par des expériences de rouge (et non par exemple par l'expérience de bleu), ce n'est pas un hasard si le danger nous est présenté par l'expérience de peur (et non par exemple par l'expérience de la colère). Enfin, il y a une contrainte épistémique. Celle-ci est révélée à la fois par les différences et les similitudes que les émotions entretiennent avec la perception. Si les émotions causent et justifient les jugements évaluatifs de façon analogue à la manière dont les perceptions causent et justifient les jugements perceptifs, les émotions entretiennent également avec leurs bases cognitives (états perceptifs, imaginatifs, doxastiques, mnésiques, etc.) d'autres rapports causaux et de justification qui les distinguent fondamentalement des perceptions et dont une théorie des émotions doit être à même de rendre compte.

\section{SENSATIONS CORPORELLES ET INTENTIONNALITÉ DES ÉMOTIONS}

Le défi consiste donc à donner une analyse des émotions qui combine de manière satisfaisante leur caractère ressenti et leur caractère intentionnel. Si nous avons jusqu'ici tenté d'expliquer en quoi les émotions devaient être conçues comme dirigées au-delà d'elles-mêmes vers des propriétés évaluatives des objets et situations, nous n'avons dit que très peu de choses sur leur dimension phénoménale. Mais à quoi fait-on plus exactement référence lorsque l'on dit que cela fait un certain effet que de ressentir des émotions?

Il semble que ressentir une émotion soit intimement lié à un certain type de ressenti corporel. Lorsque nous cherchons à décrire l'effet que cela fait que de ressentir une émotion, n'est-on pas toujours ramené à décrire la manière dont certains événements résonnent dans notre corps? Le philosophe qui a souligné avec le plus de force cet aspect crucial de la phénoménologie des émotions est bien sûr W. James. Par exemple, considérant la peur et la colère, il écrit:

What kind of emotion of fear would be left if the feeling neither of quickened heart beats
nor of shallow breathing, neither of trembling lips nor of weakened limbs, neither of
goose flesh nor of visceral stirrings, were present, it is quite impossible for me to think.
Can one fancy the state of rage and picture no ebullition in the chest, no flushing of the
face, no dilatation of the nostrils, no clenching of the teeth, no impulse to vigorous action,
but in their stead limp muscles, calm breathing and a placid face? (James, 1890: p. 379) 
A la lumière de ce passage, il convient de souligner trois points essentiels. D'abord, James critique implicitement les théories du jugement évaluatif en affirmant, tout comme l'argument de la suffisance discuté plus haut, qu'une théorie adéquate des émotions ne saurait faire l'impasse sur leur caractère ressenti. Ensuite, il associe ce caractère ressenti aux modifications corporelles qui accompagnent les émotions. Enfin, il identifie l'aspect phénoménologique des émotions au ressenti de ces modifications corporelles. Et dans cette mesure, comme le passage suivant le souligne, les émotions ne doivent pas être assimilées à des sensations brutes:

these alterations, perceived, like the original object, in as many portions of the cortex, combine with it in consciousness and transform it from an object-simply-apprehended into an object-emotionally-felt. (James, 1890: p. 474)

Les émotions sont bien plutôt des ressentis qui prennent pour objets intentionnels des modifications corporelles. Finalement, non seulement l'aspect phénoménologique des émotions est assimilé à un tel ressenti, mais les émotions ellesmêmes, et c'est là sans doute la caractéristique la plus saillante de la théorie jamesienne, sont identifiées à ces ressentis: «our feeling of the same changes as they occur IS the emotion.» (James, 1890: p. 449)

La théorie de James possède les vertus de l'élégance et de la simplicité. Avant de considérer ce qui nous paraît être ses limites évidentes eu égard à nos contraintes, deux objections souvent dirigées vers les théories d'inspiration jamesienne -et donc les théories qui vont nous intéresser dans la suite de notre discussion, doivent être brièvement levées. Ces deux objections concernent l'identification des émotions à la perception de modifications physiologiques. Cette identification requiert d'abord que des profils de modifications physiologiques typiques correspondent aux différents types d'émotions, ensuite que chaque occurrence d'émotion soit accompagnée des modifications pertinentes. Est-il vrai toutefois qu'il existe, pour chacune des émotions individuées par le langage ordinaire, de tels profils physiologiques? Et n'y a-t-il pas aussi des occurrences d'émotions sans modification physiologique d'aucune sorte? Si les réponses à ces questions ne vont pas de soi, il est loin d'être certain que l'évidence à notre disposition aille à l'encontre de la théorie jamesienne. Concernant la première objection, notez que cette théorie n'est nullement tenue de porter, comme l'objection le laisse entendre, sur tous les types d'émotions distingués par le sens commun. $\mathrm{Au}$ contraire, elle peut se contenter d'affirmer que de tels profils existent pour un certain nombre d'émotions de base, et que la différenciation des autres types d'émotions s'opère autrement, en particulier par les états cognitifs du sujet ${ }^{16}$. Par exemple, la colère aurait un profil physiologique déterminé, mais ne se distinguerait de l'indignation que dans la mesure où, dans cette dernière, le sujet conçoit l'objet de son expérience en termes moraux. Pour traiter la seconde objection, le défenseur d'une théorie jamesienne doit souligner qu'il requiert simplement l'existence d'une conscience de modifications physiologiques, que celles-ci

${ }^{16}$ Pour un développement détaillé de cette stratégie, voir Prinz (2004). 
soient présentes ou non. Des données récentes suggèrent que les mécanismes neuronaux dont la fonction consiste à détecter les modifications physiologiques peuvent être activés en leur absence ${ }^{17}$.

Si ces objections sont donc loin d'être fatales pour la théorie jamesienne, il nous faut maintenant l'évaluer à la lumière de nos contraintes. En un sens au moins, il est clair qu'elle permet de respecter à la fois l'idée que les émotions possèdent essentiellement un caractère ressenti et l'idée que celles-ci possèdent une forme d'intentionnalité. Cependant, l'intentionnalité en question permet peutêtre d'affirmer que les émotions pointent au-delà d'elles-mêmes, mais sans doute pas suffisamment loin, dans la mesure où elles ne prennent précisément jamais autre chose que le corps pour objet. Si James affirme bel et bien que «the bodily changes follow directly the perception of the exciting fact» (James, 1890: p. 449), le ressenti qui en résulte est corporel et aucunement dirigé vers le fait excitant en question. Au sens strict, donc, la peur n'est pas à propos d'une quelconque propriété du chien, la colère à propos de cette remarque, mais à propos des modifications corporelles que la cognition de ces objets engendre. En d'autres termes, la relation intentionnelle à la remarque, au chien et à leurs propriétés est épuisée par ce que nous avons appelé plus haut les bases cognitives de l'émotion. Comprise de cette manière, la théorie de James souffre donc d'un déficit évident en ne permettant pas de comprendre les émotions comme des expériences qui nous mettent en relation intentionnelle avec ce qui se trouve au delà de notre corps.

A la lumière des multiples avantages offerts par une théorie mettant ainsi l'accent sur le corps ressenti, il convient de se demander dans quelle mesure ce déficit peut être comblé. En particulier, n'y a-t-il pas moyen d'articuler la relation entre ce que James appelle «le fait excitant» et les modifications corporelles de manière à concevoir cette relation comme intentionnelle? C'est précisément le but théorique que se propose J. Prinz dans la théorie la plus aboutie tâchant de combiner les contraintes intentionnelles, phénoménologiques et épistémiques ${ }^{18}$. Prinz inscrit en effet la théorie jamesienne dans le cadre d'une conception contemporaine populaire de la représentation et de l'intentionnalité qui permet une réponse immédiate à l'objection selon laquelle les émotions jamesiennes restent confinées au corps. La stratégie est relativement simple. Elle part du constat que les modifications physiologiques qui, dans la théorie de James, constituent les émotions, covarient de manière systématique avec la présence des «faits excitants». Plus spécifiquement, et cela était déjà reconnu par James, certains types de modifications corporelles covarient systématiquement avec des types de faits excitants. Les mêmes types de faits, par exemple les perceptions d'araignées, engendrent les mêmes types de sensations corporelles et donc d'émotions, ici la peur. L'idée centrale consiste maintenant à exploiter cette relation de covariation causale systématique pour élucider la dimension représentationnelle des émotions et, ainsi, leur intentionnalité.

${ }^{17}$ Pour ces objections classiques à la théorie de James, voir Cannon (1927). Pour les réponses contemporaines esquissées ici, voir en particulier Damasio et al. (2000) et Prinz (2004), pp. 58-59.

18 Prinz (2004). 
Ceci est réalisé par l'application d'une théorie générale de la représentation selon laquelle $\mathrm{x}$ représente $\mathrm{y}$ si $\mathrm{x}$ covarie systématiquement avec y et a pour fonction de covarier de cette manière avec $\mathrm{y}^{19}$. Par exemple, il y a une relation causale systématique entre le volume occupé par un échantillon de mercure et la température ambiante. S'il fait sens de dire qu'une dilatation donnée du mercure indique une certaine température, on ne peut cependant pas encore parler de représentation, car ce n'est pas la fonction du mercure que de covarier de cette manière avec la température. Nous exploitons en revanche cette relation d'indication en fabriquant des thermomètres. La hauteur de la colonne de mercure dans un thermomètre a alors la fonction, ici assignée par nous, de représenter la température ambiante: elle la représente donc. Mais toute fonction n'est pas assignée, certaines sont naturelles. Les battements du cœur covarient systématiquement avec certains sons, mais la fonction du cœur n'est pas de produire de tels sons, mais bien plutôt de faire circuler le sang: c'est pour occuper cette fonction que cet organe a été sélectionné par l'évolution. Or, les états mentaux possèdent également des fonctions naturelles. Par exemple, il existe une covariation systématique entre certains stimuli proximaux dans le champ visuel d'un batracien et la présence de certains petits objets noirs dans son environnement immédiat. Si la covariation causale assure la présence d'une relation d'indication entre ces stimuli proximaux et la présence d'objets de ce type, le fait est que l'état perceptif a pour fonction de détecter la présence de sources de nourriture pour le batracien: pour cette raison, on considérera que ces états représentent, par exemple, les moucherons dont dépend sa survie. Cette théorie contemporaine de l'intentionnalité consiste donc à affirmer que la relation de représentation peut être intégralement élucidée en termes de relations d'indication (covariation causale systématique) et de fonction (sélection par l'évolution pour la représentation naturelle).

L'application de cette thèse générale au cas des émotions est maintenant aisée. Comme le souligne James, les sensations corporelles sont au fondement des émotions. De plus, si ces sensations ainsi que les modifications corporelles qu'elles prennent pour objet covarient avec certains types de situations, alors peut-être pourrons-nous parler des émotions comme représentant ces situations. Ceci exige bien sûr que ces émotions soient dotées de fonctions naturelles, ce qu'il n'est pas difficile d'accepter: il va de soi que les émotions sont sujettes à l'évolution par sélection naturelle, et celles-ci sont depuis Darwin et de manière aujourd'hui très répandue approchées sous l'angle de leurs fonctions adaptatives ${ }^{20}$. Et comment mieux spécifier cette fonction adaptative que par le biais de certaines propriétés communes aux situations qui engendrent ces émotions? Or, ces propriétés communes ne sont rien d'autres que les propriétés évaluatives ou objets formels des émotions que nous avons déjà rencontrés à plus d'une reprise au cours de notre discussion. La peur a pour fonction de détecter le danger et de préparer le sujet à y faire face, la jalousie a pour fonction de repérer les rivaux potentiels et le dégoût permet au sujet de réagir de manière différenciée à des substances dont la

\footnotetext{
${ }^{19}$ Cette approche de la représentation a été proposée par Dretske (1981) et affinée notamment par Millikan (1987).

${ }^{20}$ Darwin (1872), Cosmides \& Tooby (2004).
} 
proximité est néfaste. Soulignons que les fonctions des émotions peuvent également être spécifiées par le biais de leur contribution à l'organisation sociale particulière dans laquelle le sujet vit ou au sein de laquelle ses ancêtres ont été inscrits. Certains considèrent par exemple que la honte a pour fonction de signaler au sujet son statut inférieur au sein d'un groupe hiérarchique et d'indiquer au supérieur sa soumission $^{21}$. En outre, par le biais de l'apprentissage, les émotions peuvent bien sûr en venir à être déclenchées par des situations nouvelles. Ainsi, la théorie en question peut parfaitement rendre compte par exemple de la peur ressentie à la constatation du fait que ma fortune est menacée par la conjoncture économique; il suffit pour cela que les sensations corporelles propres à la peur en viennent à covarier systématiquement avec ce type de situation, parce que le sujet les appréhende comme des dangers.

Cette relecture contemporaine de la théorie jamesienne des émotions semble à première vue pouvoir respecter de manière particulièrement élégante nos trois contraintes. La contrainte phénoménologique est directement prise en compte par le recours aux sensations corporelles. Ces mêmes sensations corporelles permettent également de respecter la contrainte intentionnelle, dans la mesure où elles se voient dotées d'une fonction représentative: par leur truchement, les émotions représentent les valeurs ou objets formels des émotions. Enfin, elle respecte la contrainte épistémique: les sensations corporelles sont distinctes des faits excitants et de leur appréhension par le sujet (base cognitive de l'émotion), ce qui autorise à parler de relations causales et épistémiques entre les deux.

Mais ces contraintes sont-elles satisfaites d'une manière plausible ${ }^{22}$ ? Considérons d'abord comment cette théorie comprend la contrainte phénoménologique, à savoir le fait que la phénoménologie des émotions joue un rôle dans la présentation de la valeur au sujet. Si la théorie de Prinz fait effectivement appel à un acte intentionnel à la première personne pour rendre compte du lien entre émotions et valeurs - à savoir la perception des modifications physiologiques, peut-on faire appel à ce type de perception pour satisfaire notre contrainte? Prinz peut laisser penser que tel est le cas lorsqu'il distingue, avec Locke, contenu nominal et contenu réel et propose l'analogie suivante. Tout comme l'apparence perceptive de l'eau (contenu perceptif nominal) constitue un rapport intentionnel à ce qui s'avère être $\mathrm{H}_{2} \mathrm{O}$ (contenu perceptif réel), la conscience de certaines modifications de notre corps (contenu émotionnel nominal) constitue un rapport intentionnel à telle ou telle valeur (contenu émotionnel réel) ${ }^{23}$ ? Mais, indépendamment de la validité du programme de naturalisation de l'intentionnalité dans lequel cette affirmation s'inscrit, une chose est claire: s'il fait sens d'affirmer qu' $\mathrm{H}_{2} \mathrm{O}$ nous est donné à travers l'apparence de l'eau, il ne nous est en revanche pas donné sous cet aspect. Il n'y a rien, dans les propriétés phénoménales associées à une perception visuelle d'eau, qui rende manifeste au sujet la présence d'une substance de telle

${ }^{21}$ Voir par exemple Fessler (2004).

${ }^{22}$ Certaines des critiques qui suivent ont été développées de manière quelque peu différente dans Deonna et Teroni (2008).

${ }^{23}$ Prinz (2004: pp. 67-69). 
composition chimique. Or, l'objectif que nous poursuivons n'est-il pas de faire en sorte que la phénoménologie des émotions rende manifeste au sujet la présence d'une certaine valeur dans son environnement? A moins de nier l'une des intuitions au fondement des théories perceptives des émotions, c'est-à-dire le fait que la dimension phénoménale de l'émotion nous rend manifeste la présence d'une certaine valeur -et c'est peut-être le cas de Prinz (2004: p. 60), l'application de la distinction lockéenne pose de sérieux problèmes. Si l'on admet donc que la théorie rend compte de la dimension intentionnelle des émotions, il n'est pas évident qu'elle le fasse d'une façon qui rende justice au rôle joué par la phénoménologie des émotions dans l'accès aux valeurs.

Mais n'est-ce pas là déjà trop concéder? Dans cette théorie, les émotions sontelles réellement dans un rapport intentionnel quelconque aux valeurs? Cela est loin d'être clair si l'on fait appel aux sensations corporelles comprises comme des types de perception, dans la mesure où celles-ci sont déjà des actes intentionnels dirigés vers certaines parties du corps. L'analogie avec la perception de l'eau se trouve pour cette raison d'autant plus compromise. Dans ce dernier cas, il n'y a pas deux candidats au statut d'objet de la conscience perceptive, $\mathrm{H}_{2} \mathrm{O}$ et ce qui nous est donné visuellement, mais un seul. Ici, le lien entre l'un et l'autre est bien plutôt d'ordre constitutif. En revanche, il semble bien y avoir compétition entre deux objets au sein du type d'état mental recruté par la théorie pour fonctionner comme appréhension des valeurs. Les sensations corporelles, en effet, possèdent déjà un objet intentionnel, à savoir les parties du corps qu'elles nous font ressentir de telle ou telle manière. Et le lien entre parties du corps ressenties et valeurs n'est clairement pas d'ordre constitutif. La valeur n'est bien sûr pas aux parties ressenties du corps ce qu' $\mathrm{H}_{2} \mathrm{O}$ est aux propriétés manifestes dans la perception visuelle de l'eau. La conclusion qui s'impose alors, c'est que l'accès aux valeurs que garantit cette théorie est minimal: nous sommes, lorsque nous ressentons une émotion, conscients des modifications de notre corps, et celles-ci covarient simplement avec la présence de la valeur. Supposons que, sans que je le sache, chaque fois que l'on sonne à ma porte, ma voisine sursaute. Doit-on penser pour cette raison que ma perception auditive du carillon de ma sonnette me met en relation intentionnelle avec ma voisine? En un mot, dans la théorie jamesienne, il n'y a pas véritablement de rapport intentionnel à la valeur au sein des émotions, que celui-ci soit perceptif ou judicatif.

Si ces considérations n'étaient pas fatales à la théorie, il faut encore souligner ses implications en ce qui concerne notre contrainte épistémique. La distinction entre émotions et bases cognitives est certes respectée, mais les liens épistémiques entre émotions et jugements évaluatifs sont quant à eux plus problématiques. On se souvient qu'un atout majeur du modèle perceptif consiste à tirer profit du parallélisme manifeste entre perceptions et jugements perceptifs d'une part, émotions et jugements évaluatifs d'autre part. Dans les deux cas, l'idée est qu'un certain accès expérientiel à un type d'information justifie la formation des jugements pertinents. Or, ce parallélisme s'est ici évanoui: en effet, les émotions rendent manifestes au sujet certaines parties de son corps et non pas la présence de la valeur pertinente. Naturellement, il demeure possible d'attribuer un rôle épistémique plus indirect aux émotions par rapport aux jugements évaluatifs. Le sujet perspicace saura en temps et lieu associer la valeur pertinente à un certain type de situation qui covarie avec ce qu'il perçoit dans son corps. Mais notez que si cette 
manœuvre permet de recouvrer une partie du parallélisme, elle en manque toutefois une dimension cruciale. On est en effet obligé de doter le sujet d'une perspicacité peut-être trop grande. Ce dernier doit non seulement être capable d'associer à ce qu'il ressent maintenant dans son corps la valeur avec laquelle il sait que ce ressenti covarie, mais il doit également rattacher cette valeur à l'aspect pertinent de la base cognitive de l'émotion, par exemple tel élément d'une scène complexe perçue. Une tâche importante reste à accomplir, puisque la connexion entre propriétés évaluatives et objets perçus ne lui est pas accessible par le biais de l'émotion. Et ce dernier constat n'indique pas un biais internaliste de notre part ${ }^{24}$; même si le statut épistémique des jugements évaluatifs n'avait en dernière analyse rien à voir avec les émotions que nous citons souvent pour les justifier, une théorie satisfaisante des émotions doit pouvoir rendre compte du fait que ces expériences émotionnelles sont les raisons que nous invoquons couramment en faveur de ces jugements. Or, la façon dont cette théorie nous force à comprendre ce rapport, à savoir comme un accès intellectuel et indirect aux valeurs, est en inadéquation manifeste avec la manière ordinaire dont ces jugements sont formés. La contrainte épistémique n'est donc pas non plus respectée de manière satisfaisante.

\section{LE CORPS \\ RESSENTI COMME ACCÈS AUX VALEURS}

A ce stade, deux options s'offrent à nous. Soit nous abandonnons l'idée que le corps ressenti puisse fonctionner comme accès aux valeurs - au risque de perdre de ce fait les avantages liés au modèle expérientiel des émotions, soit nous cherchons à articuler différemment les rapports entre corps ressenti et valeurs de sorte à ce que le premier puisse directement fonctionner comme présentation des secondes $^{25}$. Mais qu'est-ce que cela peut bien signifier?

Pour commencer à répondre à cette question, rappelons-nous que l'intuition fondamentale au cœur du modèle expérientiel des émotions est que le rapport entre expériences émotionnelles et valeurs est tout sauf arbitraire. Ce n'est pas un hasard que le danger soit présenté par ce que je ressens quand j'ai peur, l'offense par ce que je ressens quand je suis en colère. C'est précisément cet aspect du problème que négligent entièrement les théories discutées jusqu'à présent. Le ressenti ne doit pas être conçu comme un signe arbitraire de la présence d'une certaine valeur, mais comme ce qui la rend manifeste au sujet. Le problème, dès lors, consiste à élucider ce que l'on veut dire, par exemple, par l'idée que le ressenti propre à la peur est, et ne peut être, qu'une présentation du danger.

${ }^{24}$ L'internalisme en épistémologie peut prendre plusieurs formes. En bref, il s'agit de la thèse selon laquelle un sujet ne peut être dit savoir que p que s'il est capable d'accéder et éventuellement de rapporter des raisons ou des justifications en faveur de $p$. Cette thèse s'oppose à l'externalisme qui soutient qu'un sujet peut savoir que $\mathrm{p}$ sans pour autant qu'il ait accès à la première personne aux justifications pertinentes.

${ }^{25}$ Bien sûr, une troisième option consiste à postuler une phénoménologie affective qui n'a rien à voir avec celle du corps et qui serait propre aux différents types d'émotions. Cette option se rapprocherait alors d'un intuitionisme à propos de notre connaissance des valeurs (e.g. Moore 1903). Cette position pose de nombreux problèmes que nous ne pouvons aborder ici. 
Notre suggestion est la suivante. Il est clair qu'au niveau phénoménologique, les propriétés évaluatives des objets ne sont pas données dans les émotions de la même manière que les propriétés visuelles ou tactiles le sont dans la vision ou le toucher. A ce niveau phénoménologique, nous sommes tentés d'affirmer que les valeurs sont appréhendées comme des aspects de l'environnement qui contraignent diversement les options comportementales à la disposition du sujet. Réfléchissons à ce en quoi pourrait consister, par exemple, une expérience de danger. Que dire d'autre si ce n'est que le danger d'une certaine situation m'est rendu manifeste par le fait que celle-ci m'est présentée comme réclamant un comportement propre à neutraliser certains de ses aspects? Et il nous semble que nous venons précisément de décrire ce dont nous faisons l'expérience dans la peur. Dans cette expérience, nous ressentons notre corps comme mobilisé en vue d'une neutralisation de ce type, quelle que soit la forme plus spécifique qu'elle prenne dans des circonstances données. L'aspect fondamental de cette approche consiste à considérer que, dans les émotions, le corps est ressenti comme mobilisé en vue d'actions potentielles et que la manière spécifique dont il est mobilisé s'interprète tout naturellement comme appréhension de certaines parties de l'environnement sous leurs aspects évaluatifs. Ici, donc, les sensations corporelles ne sont pas comprises comme de simples effets de cognitions de l'environnement, elles en viennent à jouer directement, en vertu de leur phénoménologie, un rôle de présentation intentionnelle de leurs objets propres, à savoir les valeurs. En un mot, la seule solution viable, dans un cadre théorique où les sensations corporelles sont recrutées pour rendre compte de l'intentionnalité des émotions, consiste à comprendre celles-ci comme des ressentis du corps mobilisé pour l'action ${ }^{26}$ : c'est dans ce sens précis que l'on peut dire qu'une émotion est une expérience de valeur.

A supposer que cette théorie soit viable, elle permet non seulement de prendre en compte les motivations initiales pour un modèle perceptif des émotions, mais en outre de satisfaire nos trois contraintes. Les émotions sont conçues comme des états mentaux dirigés vers des valeurs (contrainte intentionnelle); ces valeurs sont comprises comme étant rendues manifestes par la phénoménologie corporelle propre aux différents types d'émotions (contrainte phénoménologique); enfin, les ressentis corporels étant indépendants des bases cognitives des émotions, la théorie permet potentiellement d'articuler de manière élégante les rapports causaux et épistémiques entre bases cognitives, émotions et jugements évaluatifs (contrainte épistémique). En particulier, si l'expérience émotionnelle est en ellemême une expérience en première personne d'une propriété évaluative d'un objet ou d'une situation, alors cette expérience devrait pouvoir compter comme raison pour le sujet de juger que l'objet ou la situation instancie bel et bien cette valeur.

La viabilité de cette approche dépend naturellement, et en premier lieu, de la possibilité de l'étendre à de nombreux types d'émotions. Est-il plausible d'affirmer qu'aux différents types d'émotions correspondent différents profils ressentis du corps mobilisé vers l'action ou, pour être plus précis, peut-on appliquer cette approche aux émotions de base? Le dégoût par exemple doit être compris dans le

${ }^{26}$ La richesse des formes que peut prendre la préparation à l'action a été particulièrement mise en évidence par Frijda $(1986,2006)$. 
cadre de cette approche comme l'expérience d'un objet, typiquement de nature organique, comme réclamant une mise à distance par «obturation» des sens. Dans le cas de la colère, le corps est mobilisé en vue d'une forme d'assaut de l'objet et, dans l'affection, mobilisé en vue d'une forme de cajolerie.

L'approche semble donc à première vue pouvoir s'étendre à plusieurs types d'émotions. Il nous faut pourtant mesurer ses implications. D'abord, comme toutes les théories d'inspiration jamesienne, elle requiert qu'aux différents types d'émotions correspondent des profils de modifications physiologiques distincts. De même, elle implique que toute occurrence d'émotion s'accompagne d'une conscience de ces modifications. Nous avons déjà eu l'occasion de souligner la manière dont ces théories pouvaient satisfaire ces exigences.

Deux difficultés spécifiques à la théorie ici suggérée doivent toutefois être considérées. Premièrement, il semblera étonnant à beaucoup que les sensations corporelles soient dites dirigées vers autre chose que des parties du corps. Ensuite, étant donné l'accent mis sur les tendances à l'action, il n'est point évident que l'analyse puisse s'appliquer aux nombreuses émotions qui n'ont pas de lien direct avec l'action.

Commençons par cette dernière difficulté. Par exemple, que nous présente le corps ressenti dans la tristesse ou dans l'admiration? L'une et l'autre semblent au moins à première vue indépendantes de toute conscience d'une possibilité quelconque d'action. A y regarder de plus près, cependant, les choses ne sont pas aussi évidentes. Dans la tristesse, l'abattement du corps n'est-il pas précisément ce dont nous avons besoin pour qualifier la manière dont un objet est appréhendé comme une perte? L'expérience de la perte n'est-elle pas précisément la conscience de la privation de certaines possibilités d'action par rapport à un objet donné? Et est-il vrai qu' aucune tendance à l'action n'est associée à l'admiration? Rien n'est moins sûr. A nos yeux, il fait sens d'affirmer que faire l'expérience d'un objet comme élégant, charmant ou beau consiste à l'appréhender comme demandant une attention perceptive soutenue d'un certain type, l'explorer du regard par exemple.

En ce qui concerne la première difficulté, il faut répondre de la manière suivante. Les sensations corporelles pertinentes ne concernent les parties du corps que lorsque l'attention est dirigée directement vers ces sensations. Or, cela n'est précisément pas le cas lorsque nos émotions répondent aux saillances de notre environnement. Dans ces cas, bien au contraire, le corps est ressenti comme dirigé vers certains aspects d'une situation donnée. La conscience des tendances à l'action, en particulier, concerne uniquement les possibilités d'agir par rapport à certains objets ou situations. C'est seulement lors d'une redirection de l'attention - ce qui arrive fréquemment et en particulier lorsqu'aucune action ne s'impose naturellement au sujet, ou après l'action en question, que l'attention peut se porter sur les parties du corps concernées. Par exemple, je sais que je dois rester immobile pour éviter d'exciter l'ours qui me fait face, ce qui me donne l'occasion de prendre conscience des battements de mon cœur et de ma tension musculaire. De même, si l'ours décide par chance de m'épargner, la tension antérieure de mon corps se révèle dans sa disparition progressive. Avant cela, l'expérience affective était entièrement dirigée vers le danger représenté par l'ours, à savoir comme demandant une certaine réponse de neutralisation des aspects pertinents de la situation. En un mot, il faut distinguer ici une conscience «naïve » du corps, entièrement dirigée vers l'extérieur par le biais de la conscience des tendances à 
l'action, d'une conscience réflexive dirigée vers le corps plutôt que vers ce quoi il tend. Il semble donc qu'aucune des deux difficultés principales soulevées plus haut à l'encontre de notre approche ne soit insurmontable.

Plus généralement, la viabilité d'une conception expérientielle des émotions par le biais du corps ressenti dépend de la possibilité de traduire le type d'expérience propre à chaque émotion de base en terme de mobilisation ressentie du corps en vue de l'action. Si cela s'avérait possible, et nous pensons qu'un certain optimisme est de rigueur, alors le rôle du corps au sein des émotions est non seulement compatible avec une forme minimale de rationalité des émotions, il en constitue même le fondement. La mobilisation ressentie du corps nous présente les valeurs, et ce type d'expérience cause et nous donne des raisons en faveur de certains jugements évaluatifs.

Ecole polytechnique fédérale, Lausanne

Université de Genève

\section{BIBLIOGRAPHIE}

Armstrong, D. M. (1968/1993). A Materialist Theory of the Mind, Routledge.

Cannon, W. B. (1927). «The James-Lange Theory of Emotions: A Critical Examination and an Alternative Theory », American Journal of Psychology, vol. 39, pp. 106-124.

Colombetti, G. (2005). «Appraising Valence», Journal of Consciousness Studies 12.8-10, pp. 103126.

Cosmides, L. et Tooby, J. (2004). «Evolutionary Psychology and the Emotions », in Michael Lewis et Jeannette M. Haviland-Jones (dir.), Handbook of Emotions, Guilford Press.

Crane, T. (1992). «The Nonconceptual Content of Experience», in T. Crane (dir.), The Contents of Experience, Cambridge: Cambridge University Press.

De Sousa, R. (1987). The Rationality of Emotions. Cambridge: MIT Press.

Damasio, A. R. (1994). Descartes' Error: Emotion, Reason and the Human Brain. New York: Putnam.

Damasio, A. R., Grabowski, T.J., Bechara, A., Damasio, H., Ponto, L. L. B., Parvizi, J. et Hichwaet, R. D. (2000). «Subcortical and Cortical Brain Activity during the Feeling of Self-Generated Emotions », Nature Neuroscience 3.10, pp. 1049-1056.

Darwin, C. (1872/1998). The Expression of the Emotions in Man and Animals, Harper Collins Publisher.

Deigh, J. (1994). «Cognitivism in the Theory of Emotions», Ethics 104.4, pp. 824-854.

Deonna, J. A. (2006). «Emotion, Perception and Perspective», dialectica vol. 60.1, pp. 29-46.

Deonna, J. A. et Teroni, F. (2008). Qu'est-ce qu'une emotion, Vrin.

Döring, S. (2007). «Seeing what to do: Affective Perception and Rational Motivation», dialectica 61.3, pp. 363-394.

Dretske, F. (1981). Knowledge and the Flow of Information, MIT Press.

Fessler, D. M. T. (2004). «Shame in Two Cultures: Implications for Evolutionary Approaches», Journal of Cognition and Culture 4.2, 207-262.

Frijda N. H. (1986). The Emotions, Cambridge, Cambridge University Press.

Frijda, N. H. (2006). The Laws of Emotion, Lawrence Erlbaum.

Goldie, P. (2000). The Emotions. A Philosophical Exploration, Oxford, Oxford University Press.

Goldie, P. (2004). «Emotion, Feeling and Knowledge of the World», in R. C. Solomon (dir.), Thinking about Feeling, Oxford University Press. 
James, W. (1890/1950). The Principles of Psychology, Dover.

Jones, K. (2006). "Quick and Smart? Modularity and the Pro-emotion Consensus», Canadian Journal of Philosophy, 36, pp. 3-27.

Kenny, A. (1963). Action, Emotion and the Will, London, Routledge and Kegan Paul.

Livet, Pierre (2002). Emotions et rationalité morale, PUF.

Millikan, R. G. (1987). Language, Thought and Other Biological Categories, MIT Press.

Moore, G. E. (1903/1988). Principia Ethica, Prometheus Books.

Mulligan, K. (1998). «From Appropriate Emotions to Values», The Monist 81, pp. 161-88.

Nussbaum, M. C. (2001). Upheavals of Thought. Cambridge University Press.

Prinz, J. (2004). Gut Reactions: A Perceptual Theory of the Emotions, Oxford University Press.

Roberts, R. C. (2003). Emotions: An Essay in Aid of Moral Psychology. Cambridge University Press.

Solomon, R. C. (1988). «On Emotions as Judgements», American Philosophical Quarterly, 25.2, pp. 183-191.

Tappolet, C. (2000). Emotions et Valeurs, Paris, Presses Universitaires de France.

Teroni, F. (2007). «Emotions and Formal Objects», dialectica, 61.3, pp. 395-415.

Tye, M. (2002). «Representationalism and the Transparency of Experience», Nous 36.1, pp. 137-151.

Tye, M. (2008). «The Experience of Emotion: An Intentionalist Theory», Revue internationale de philosophie. 